\title{
Study of a half small scale quadricopter balance: illustration of a complex analogue electronic feed back
}

\author{
Ph.DONDON(1) -J.M.MICOULEAU(1)-J.LEGALL(1) - A.DEOLA(2) \\ (1)Université de Bordeaux IPB UMR 5818, Av Dr. A. Schweitzer 33405 Talence, FRANCE. \\ (2) Université de Bordeaux, IUT GEII, 15 rue Naudet 33175 Gradignan, FRANCE
}

\begin{abstract}
This paper presents the study and the realization of an electronic analogue feed back stabilisation system for a small scale "half-quadricopter". Such flying objects are now well-known and many commercial systems are available. However, they look as full digital embedded "black boxes". The originality of our work stands in the practical approach, "intuitive" understanding and the analogue electronic COTS design of a complex system. Moreover, this subject is a concrete opportunity to illustrate the feedback major concepts. We give first the context of the study and the mains specifications. Available hardware (mechanical parts, sensors) are briefly described. After characterisations, modelling and simulations are presented. Some experimental results are given before concluding.
\end{abstract}

Key words: Circuit and system modelling and simulation, analogue feedback, UAV brushless motor control.

Received: December 12, 2019. Revised: May 22, 2020. Accepted: May 27, 2020. Published: June 8, 2020

\section{Introduction}

\subsection{Generalities}

Small scale quadricopters quadricopter (also called UAV) are well known since a long time. Extremely sophisticated models [1] with remote I-phone control and embedded cameras are available in commercial shops. Annual contests are organized in many countries. However, building and designing such flying objects still remains a technical "adventure" and a team challenge. It requires many complementary competencies, curiosity and "up to date" electronic knowledge. This kind of project merges research aspects and didactical interests.

Design of quadricopter is generally based on a mathematical modelisation and 3D state equations [2], [3], [4], [5]. Flight is controlled by a embedded real time algorithm including Kalman filters [7].

\subsection{Justification of our study}

Around half of population is "left brain" while the other is more "right brain". It means that in researcher's world, half of them are pure mathematical and abstract brain, and half of them have a more sensitive, artistic brain. This repartition is known from Hermann diagram [8]. Thus, for those who do not have a pure "mathematical mind", it is quite difficult to understand how to program abstract equations and impact of parameters on the flight performances.

The aim and originality of the presented paper is first to understand by a practical and experimental approach the different aspects of UAV control, the impact of disturbances and so on. Secondly, we choose to make an analogue design for a more intuitive understanding of physical phenomenon which are obviously analogues.

Finally, a "bottom-up" strategy allows to go to theory with a better control and better knowledge of parameters adjustment effects, flight stability...

\section{Description of the project}

\subsection{Aim of the project}

The final aim of this project is to design an embedded electronic board to drive as simple as possible an autonomous small scale quadricopter: A pilot will drive the UAV with a commercial standard $\mathrm{RC}$ remote control $40 \mathrm{MHz}$.

Firstly, study starts by analysis, understanding and design of one axis (rolling or pitching) balance control system. Even if the complexity of the presented design is far from the reality [9], [10], [11], 
[12], it is enough to deal and to understand the major principles of feed back control effect of non linearity, and to get a right idea of sophisticated sensors uses.

\subsection{Technical specifications}

The main electronic specifications are:

- General power supply: 11VDC, 2700mAh LiPo battery cell,

- Electronic board power supply 5VDC

- Accelerometer/inclinometer: MEMS Freescale inc.

- Gyro module: MEMS Invensense inc.

- Brushless DC engines, propeller: 20cm diameter

- Quadricopter diameter: \# 50cm

- Remote control (not used for this first part of study): 40MHz FM, 4 channels:

- Vertical motion control

- Left /Right motion control

- Front /Back motion control

- Lace motion control

The design must be as well as possible analogue to analyze easily electrical signals and to understand finely the effect of each electronic block.

\subsection{Main flying parameters definition}

The full control of the UAV requires four channels corresponding to the four basic necessary movements of the quadricopter as described in figure 1 to 4 .

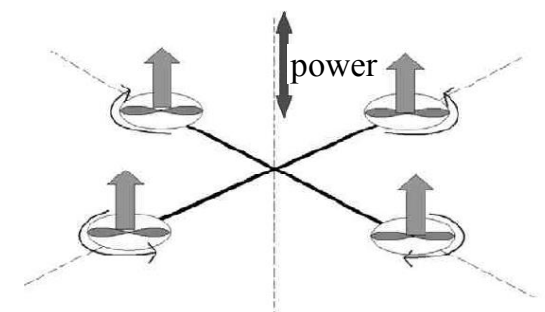

Figure 1: Vertical thrust (altitude control) (the same power is applied to the four motors)

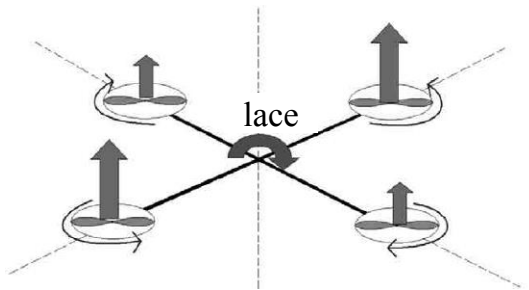

Figure 2: Lace control (rotation on itself)

(The same amount of power is applied to the two opposite motors.)

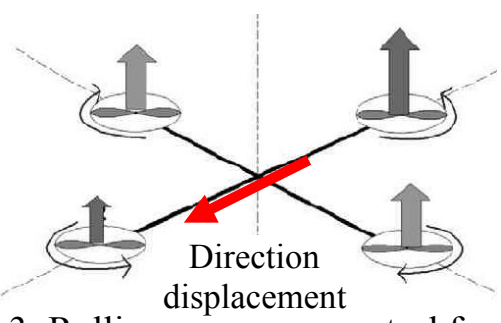

Figure 3: Rolling (airection control front/back) (Increase of the power on one of the opposite motors)

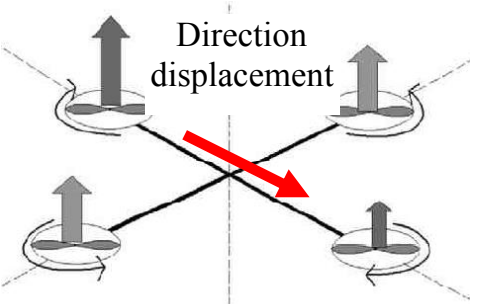

Figure 4: Pitching (direction control left/right) (Increase of the power one of the opposite motors on the second arm)

The propellers are turning clockwise and counter clockwise two by two, in order to suppress auto gyration phenomenon.

\section{Short hardware description}

\subsection{Three axis accelerometer/inclinometer module}

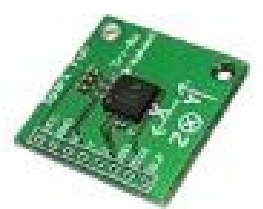

Figure 4: Accelerometer module "MMA7260Q" de Freescale

Among available sophisticated sensors [13], [14], [15], we use a simple integrated three axis accelerometer/inclinometer with an analogue output interface, from Freescale company [16].

\subsection{Brushless motors and control}

We chose four BLDC motor X-BL52S from Hacker Company dedicated to X-UFO flight and their integrated BLDC 3 phases controllers (ref. EK10350). These integrated BLDC controllers are based 
on back electromotive force (EMF) measurement instead of the Hall sensors. That is a "sensorless" control [17], [18].

The rotational speed depends on the frequency of voltage waveforms across the three phases. It is controlled by a BLDC controller input and a PWM $20 \mathrm{~ms}$ period input signal. Speed varies from 0 to maximum when the pulse width of the control signal varies from $1 \mathrm{~ms}$ to $2 \mathrm{~ms}$ according to the servo control hobbyist standards.

\subsection{Mechanical frame}

This first "low cost" test frame is made of two perpendicular wood arms. Weight of mechanical frame and motor is $290 \mathrm{~g}$. We add a LIPO $190 \mathrm{~g}$ battery cells and electronic boards $40 \mathrm{~g}$. Thus the total weight is around $520 \mathrm{~g}$ full compatible with the total thrust given by the four propellers.

\subsection{General synoptic}

The final whole schematic diagram will be as given in figure 5. Red path shows the feed back loop presented in this study, for horizontal stabilisation on one axis.

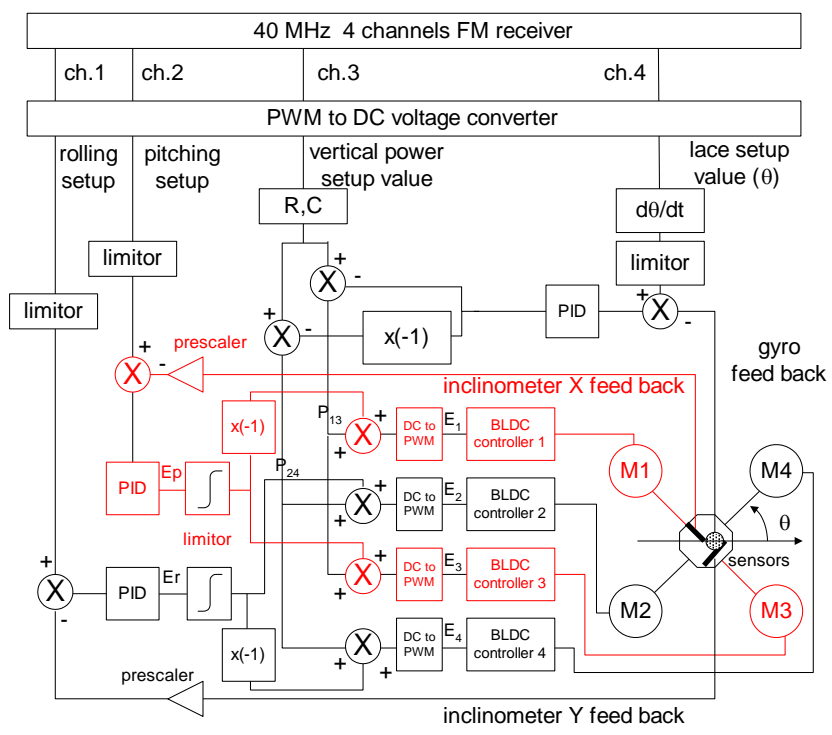

Figure 5: Quadricopter general block diagram

In this study, remote control is not used: Pitching and rolling set up control values are done by simple potentiometers which generates adjustable 0-5V DC voltages.

\section{Half quadricopter characterisation}

As told before, most of the cases and examples found in literature shows that the modelling of quadricopter is generally obtained from mathematical state and coupled movement equations [18], [19], [20]. They are entered in software such as MATLAB in order to generate an algorithm implemented in a processor. Our aim is to propose another approach more practical to facilitate the initial understanding of physical aspects and to illustrate the electronic feed back concept. Thus, this approach requires an experimental characterisation of the main parts of the system. Then, a behaviour modelling of the mechanical and electronic system will be extracted in order to design the stability control system.

\subsection{Inclinometer module characterisation}

Firstly, the sensor is characterised as follow: the reference angle at $0^{\circ}$ correspond to the horizontal position. $\theta$ angle is oriented as indicated in figure 6 .

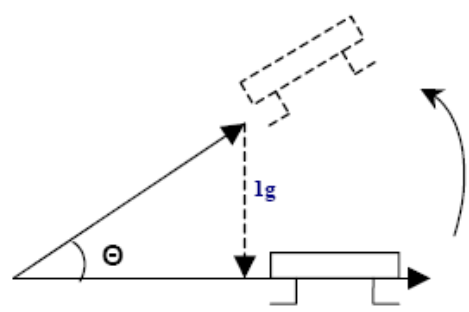

Figure 6: inclination angle $\theta$

From experimental test, we obtain the static relation between the inclinometer output DC voltage and the angle $\theta$ (cf. figure 7).

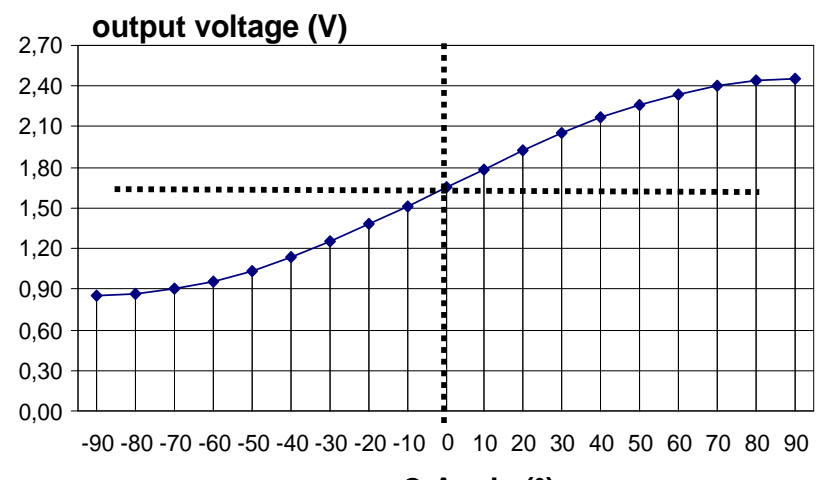

$\Theta$ Angle ( $\left(^{\circ}\right)$

Figure 7: Calibration curve MMA7260Q 
From this result we get the following equation:

$$
\mathrm{V}_{\text {OUT }}(\mathrm{mV})=1650 \mathrm{mV}+800 \mathrm{mV} \times \sin \theta
$$

Under normal flight conditions, $\theta$ should be smaller than $15^{\circ}$. Thus, the equation can be linearised:

$$
\mathrm{V}_{\text {OUT }}(\mathrm{mV})=1650 \mathrm{mV}+800 \mathrm{mV} \times \theta
$$

From a dynamic point of view, the sensor is a first order system and the cut-off frequency is around $800 \mathrm{~Hz}$

\subsection{Vertical thrust characterisation}

\subsubsection{Static characterisation}

For this purpose, we use a hand made test equipment (figure 8):

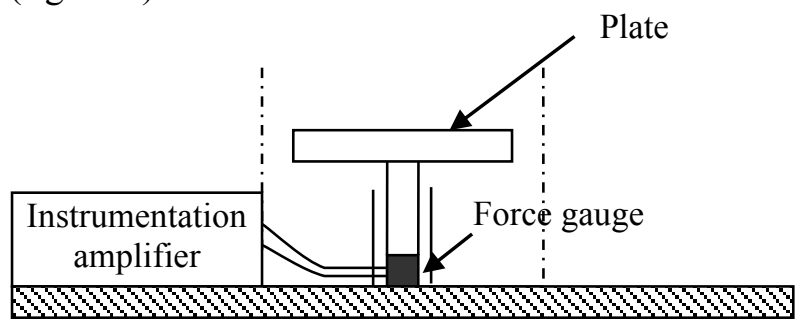

Figure 8: Experimental test bench view

It consists of a Honeywell FSG15N1A Wheatstone bridge force gauge associated to an integrated instrumentation amplifier AMP04 (PMI) supplied under $5 \mathrm{~V}$. The DC output voltage is directly function of the vertical force applied on the sensor. Using a set of calibrated weight (1gr to 500gr), our measurement system was firstly calibrated (static and dynamic).

Then, one of the 4 BLDC engines, ballasted of $500 \mathrm{gr}$ with its propeller is fixed with its BLDC speed PWM controller (figure 9).

Force sensor excluded, the space under the propeller remains free to avoid parasitic modifications of thrust and turbulences. Propeller is high enough to avoid floor effect.

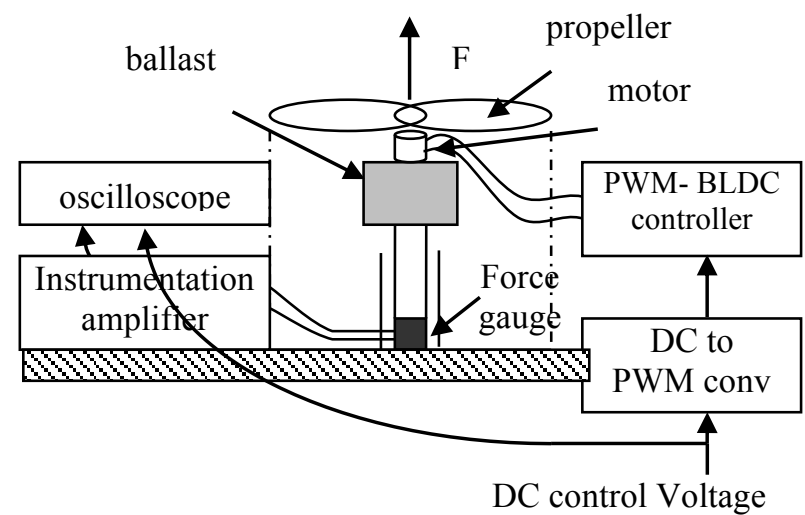

Figure 9: Static and dynamic vertical push test

When the propeller turns, a vertical push $\vec{F}$ is generated. One reads directly on the output force gauge the corresponding lightening. Thus, we can then deduce the static characteristic of the vertical force intensity versus the DC control voltage applied to the BLC controller.

Several tests, (increasing and decreasing successively the control signal) have been performed to obtain an average response given in figure 10: It must be mentioned that "Vertical push" vs. "DC control voltage" is not a linear function.

Knowing the weight of the quadcopter, it is easy to determine the minimum required control voltage value to raise the UFO.

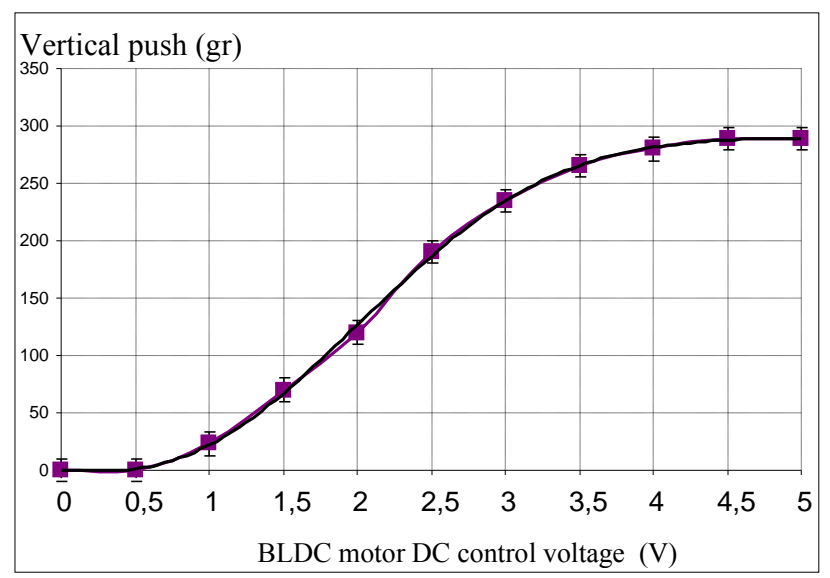

Figure 10: Average vertical push vs. input control DC control voltage

\subsubsection{Dynamic thrust characterisation}

The BLC DC engine, ballasted of $500 \mathrm{~g}$ is always fixed on the same test apparatus. A voltage pulse is applied to the speed controller input. 
Due to parasitic mechanical vibrations, we obtained the noisy response in figure 11. And the rebuilt doted line gives the correct response.

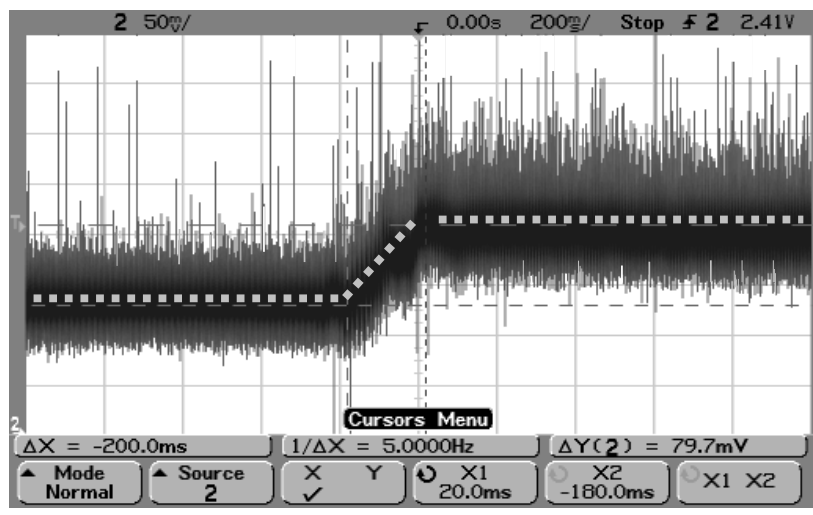

Figure 11: Thrust dynamic pulse response

Trace 1: Output voltage response to an input control voltage pulse (PWM step from $1,3 \mathrm{~ms}$ to $1,6 \mathrm{~ms}$ ) Horizontal scale: $200 \mathrm{~ms} / \mathrm{div}$

Vertical scale: $50 \mathrm{mV} / \mathrm{div}$

From the ramp response, equivalent transfer function vertical push vs. DC control voltage looks like a pure integrator $(1 / p)$.

\subsection{Pitching and/or rolling movement full characterisation}

In order to extract a modelling pitching or rolling movement must be characterized. Thus, we will obtain a global response including electronic DC to PWM converter, BLDC controller, motor, propeller, and inclinometer.

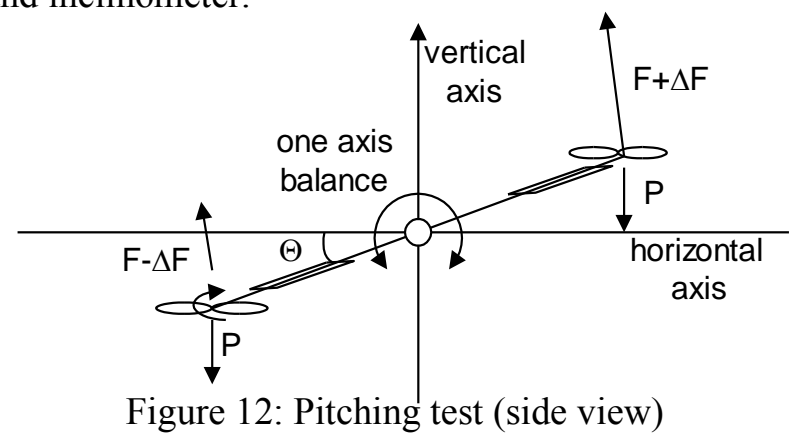

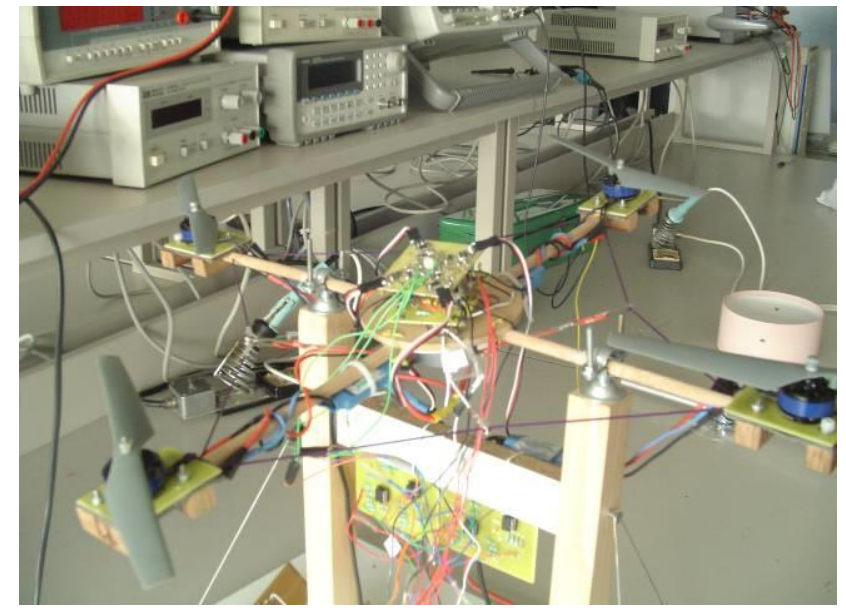

Figure 13: Pitching characterisation test bench

Figure 13 shows the experimental test bench for the characterisation of the rolling transfer function. Ball bearing has been placed on the locked axis to reduce the friction and to make the rolling movement as "free" as possible. A DC control voltage is applied to put the rolling arm horizontal. An AC voltage is superposed to this DC static point. Quadricopter oscillates around the horizontal position and the inclinometer gives an AC output voltage (image of $\Theta$ angle) which is registered to obtain the open loop response.

This experimental extraction (Figure 14) is extremely difficult for many reasons:

- Due to slow movements of the quadricopter, the working frequency range is extremely low $(10 \mathrm{mHz}$ to $100 \mathrm{~Hz}$ ).

- The variations of angle $\Theta$ must be small, (less than 15 degrees) to respect as well as possible a "linear" behaviour.

- Quadricopter is obviously not under true flying conditions (friction of the arms, flexibility of connexions wires...)

- High speed rotation of the BLDC motors generates mechanical vibrations at $1.2 \mathrm{kHz}$ transmitted to the wood arms, towards sensors: Vibration amplitude increases with rotation speed. Thus, an external low pass filter $100 \mathrm{~Hz}$ has been added to reject noise from inclinometer output voltage.

Even if we obtain an approximated transfer function, it allows obtaining basic information required for loop correction determination. 


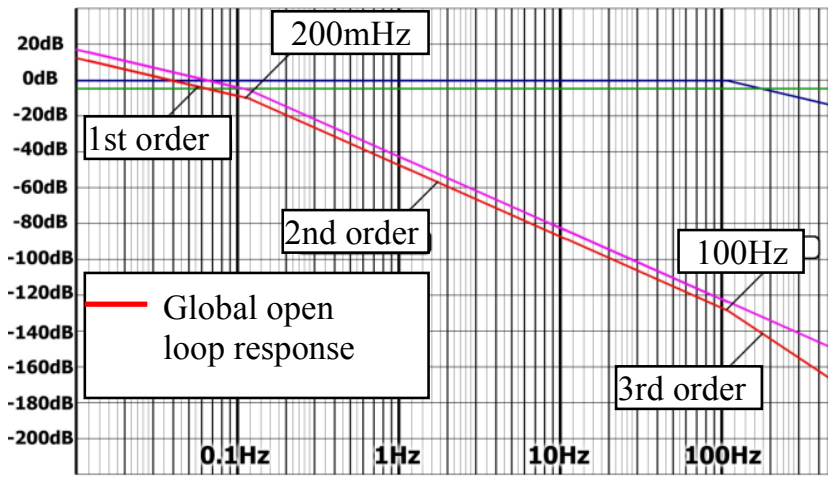

Figure 14: Experimental open loop transfer function (pitching)

\section{One axis quadricopter modelling}

From theses characterisations, behaviour modelling and a simplified transfer function $\mathrm{A}(\mathrm{p})$ is extracted for future simulations as indicated in figure 15.

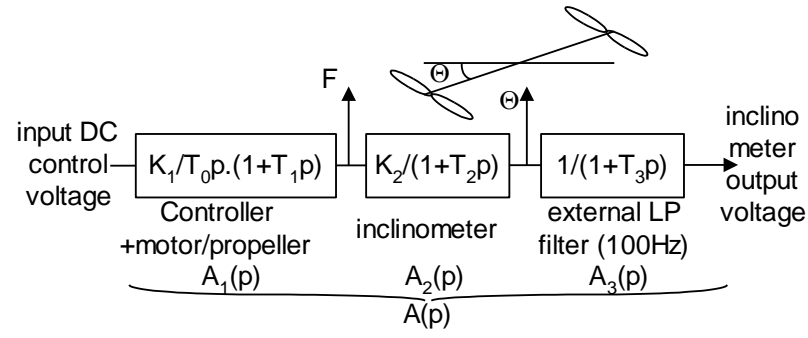

Figure 15: pitching (or rolling) behaviour modelling

Where: $F_{0}=1 / 2 \pi . T_{0} \# 100 \mathrm{mHz}, F_{1}=1 / 2 \pi . T_{1} \# 200 \mathrm{mHz}$ $\mathrm{F}_{2}=1 / 2 \pi . \mathrm{T}_{2} \# 800 \mathrm{~Hz}$ and $\mathrm{F}_{3}=1 / 2 \pi . \mathrm{T}_{3} \# 100 \mathrm{~Hz}$

These very low cut-off frequencies are obviously due to mechanical inertia.

\section{Analogue Feed back loop design}

The aim of this preliminary design is thus to control horizontality of the quadricopter on one axis (pitching or rolling). We have to design a system able to control the angle of inclination $\Theta$ with horizontal plane, by a set up control value, represented here by a DC control voltage. It is adjusted by a simple potentiometer. For a first and simplified approach, interaction and coupling between the four different flying parameters control values is not take into account. We consider only balance solving, as a single variable one axis problem.

\subsection{Brushless motor control}

The user control input of an integrated BLDC back electromotive force controller consists of a typical PWM signal as $(20 \mathrm{~ms}$ period, pulse width variable from $1 \mathrm{~ms}$ to $2 \mathrm{~ms}$ ). The rotational speed depends on the pulse width. Thus, an analogue board to convert a simple 0-5VDC control voltage into a $1 \mathrm{~ms}-2 \mathrm{~ms}$ PWM signal was designed (figure 16).

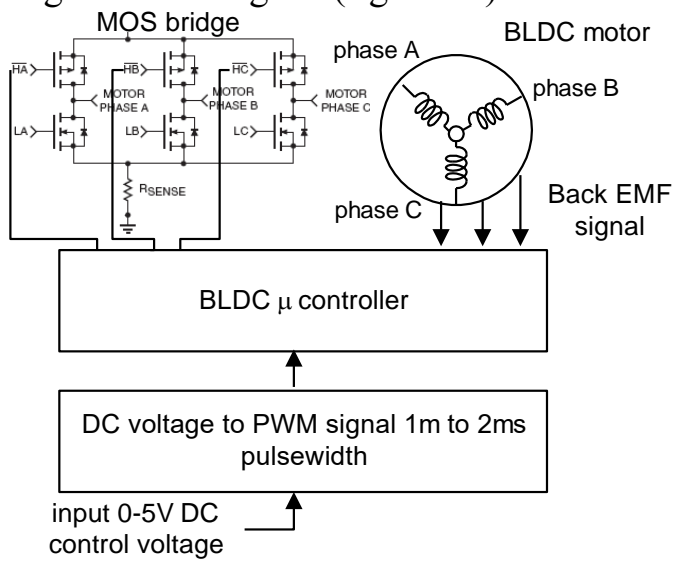

Figure 16: Brushless motor control

This DC to PWM conversion uses LMC6482 and MAX 4291 Rail-To-Rail Operational amplifiers and classical NE555 in Surface Mounted Technology for weight and size reasons.

\subsection{One axis feed back loop block diagram}

The general block diagram of the design is given in figure 17. M1 to M3 represents the two identical BLDC motors:

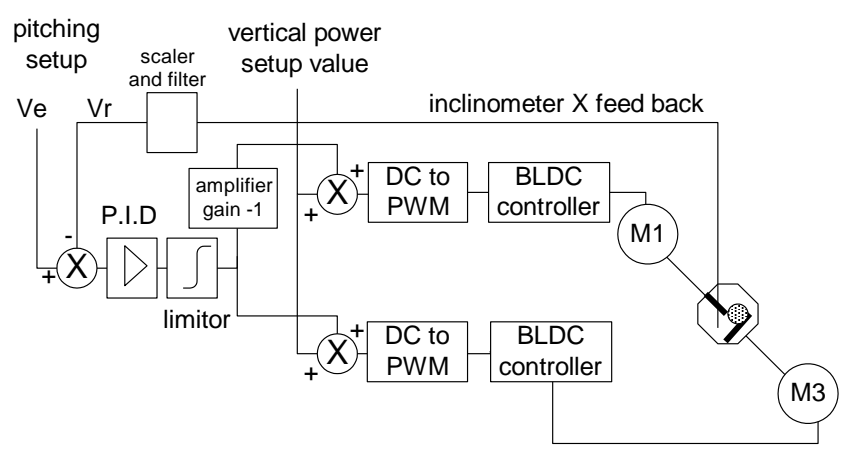

Figure 17: One axis feed back loop 
- The DC voltage given by set up potentiometer (which represents a future joystick position is compared to returned value,

-After PID for correction, the vertical push set up value is added to rolling set up value,

- Resulting voltage is converted in PWM signal, applied to BLDC controllers,

- The boxes " $\mathrm{x}(-1)$ " represent a unit negative gain amplifier to balance the movement: Action is done on both M1 and M3 opposite motors: we increase the power on one motor while the power is lowered on the other one.

- Inclinometer output is filtered, scaled and amplified and compared to set up value.

In the loop, we added voltage limiters to avoid excessive movements. The two control loops "pitching" and "rolling" are identical.

\subsection{Localisation of disturbances inputs}

In the classical feedback theoretical courses, parasitic signals are schematized on a general block diagram as indicate on figure 18a. And it is mentioned that one of the interests of a feed back loop is to reduce the effect of disturbances.

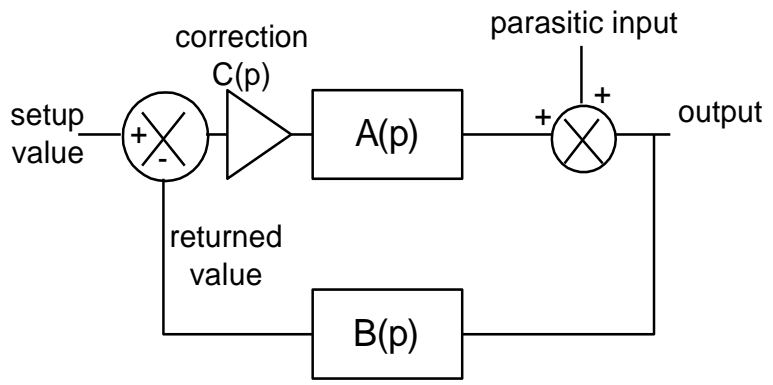

Figure 18a: Feedback general block diagram

Here, these basic aspects of feed back have to be highlighted and refined. Indeed, reducing wind disturbance, shocks and/or vibrations effects is very important for the stability of the quadricopter.

But when $A(p)$ consists of several blocks $A_{1}, A_{2}, A_{3}$ (like described in figure 15), where are exactly entering the parasitic signals in the loop? Here, for example, the wind will act on inclination but between which sub-blocks of $A(p)$ can we place this input? The same questioning happens for mechanical wood frame vibrations.
These data are obviously required to simulate correctly the loop behaviour and to compute P.I.D corrector.

As wind or shock impacts the angle inclination $\Theta$ of the half-quadricopter like the DC control signal, this input will be placed at the input of $\mathrm{A}(\mathrm{p})$ block.

As the mechanical frame vibrations only generates noise on sensor output, without changing inclination, this input is added between the first and second block of $\mathrm{A}(\mathrm{p})$ at the inclinometer input. Thus, the final schematic diagram is indicated in figure $18 \mathrm{~b}$.

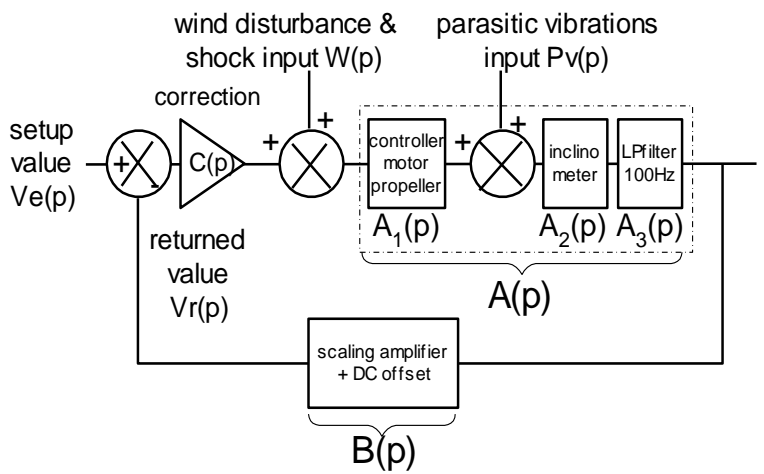

Figure 18b: Disturbances input location

The corresponding global transfer function in closed loop is (3):

$\operatorname{Vr}(\mathrm{p})=\operatorname{Ve}(\mathrm{p}) \cdot \frac{\mathrm{C}(\mathrm{p}) \cdot \mathrm{A}(\mathrm{p}) \mathrm{B}(\mathrm{p})}{1+\mathrm{C}(\mathrm{p}) \cdot \mathrm{A}(\mathrm{p}) \cdot \mathrm{B}(\mathrm{p})}+\operatorname{Pv}(\mathrm{p}) \cdot \frac{\mathrm{A}_{1}(\mathrm{p}) \cdot \mathrm{A}_{2}(\mathrm{p}) \cdot \mathrm{B}(\mathrm{p})}{1+\mathrm{C}(\mathrm{p}) \cdot \mathrm{A}(\mathrm{p}) \cdot \mathrm{B}(\mathrm{p})}+\mathrm{W}(\mathrm{p}) \cdot \frac{\mathrm{A}(\mathrm{p}) \cdot \mathrm{B}(\mathrm{p})}{1+\mathrm{C}(\mathrm{p}) \cdot \mathrm{A}(\mathrm{p}) \mathrm{B}(\mathrm{p})}$

As this function is quite complex, its behaviour is studied by Spice simulations as indicated below.

\section{SPICE modelling and simulation}

\subsection{Open loop schematic and simulation}

Once characterisation performed and modelling extracted, we can now study and predict the behaviour of the one axis feed back loop control system by Spice simulations. The mixed (electrical and analogue behaviour) ORCAD- SPICE schematic corresponding to the open loop system is given in figure 19. It includes the equivalent schematic previously given in figure 15 , voltage limiter, a non linear Spice component simulating inclinometer saturation, post-sensor amplifier and the two additional disturbance inputs.

Figure 20 gives the simulated response of the modelled system. Comparison with the $3^{\text {rd }}$ order 
experimental curve of figure 14, shows a correct matching.

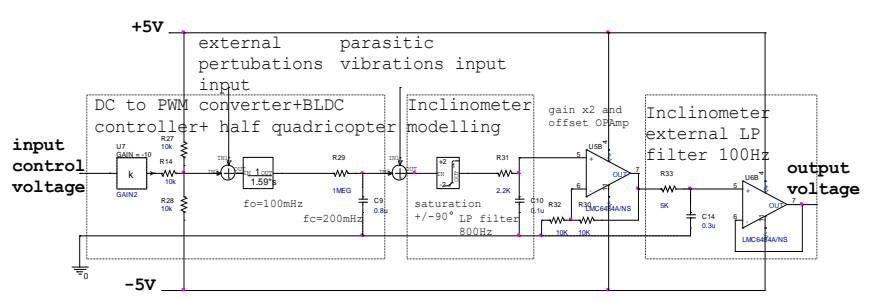

Figure 19: Open loop schematic

Gain $(\mathrm{dB})$

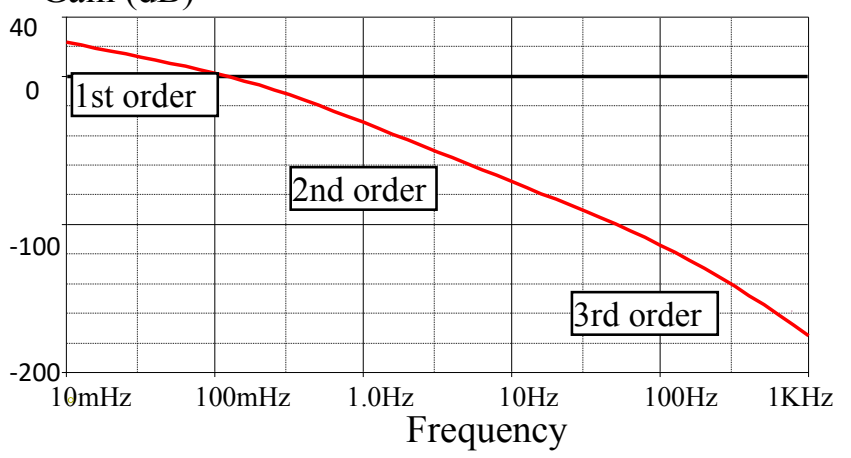

Figure 20: Simulated open loop gain

\subsection{Proportional, Integral, Derivative correction (P.I.D)}

\subsubsection{Understanding correction necessity}

An intuitive approach can be done by analysing main tendencies and effects of each input on the loop behaviour:

- A(p) has an intrinsic integration behaviour in low frequencies. Thus, static error is naturally cancelled regarding the set up control input $\mathrm{Ve}(\mathrm{p})$.

- Regarding input W(p) (wind, permanent weight difference between arm extremities, shocks), and relation between $\mathrm{W}(\mathrm{p})$ and $\operatorname{Vr}(\mathrm{p})$ in equation (3), a integral correction is required to cancel effect of DC and low frequencies disturbances. For that purpose, a pure integrator stage with OP amp is needed. It must be located obligatory before the $\mathrm{A}(\mathrm{p})$ input.

- Then, regarding the open loop order, a derivative correction is needed for frequencies above $200 \mathrm{mHz}$. A full derivative effect till the high frequencies should be perfect. Unfortunately, the Gain $\mathrm{x}$ Bandwidth product of the used OP amp $(1 \mathrm{MHz})$, limits the derivative effect, creates a cut off frequency and adds a supplementary order in the loop (see figure 21 and 22).
- At last, a low pass behaviour above $100 \mathrm{~Hz}$ is required to reject vibrations from the $\operatorname{Pv}(p)$ input disturbances.

Thus, such integral derivative correction makes a 1 st order frequency small range between two $2^{\text {nd }}$ order segments on the open loop function:

- Static gain must be finely adjusted between a minimum and a maximum value, to make this first order segment crossing the critical $0 \mathrm{~dB}$ point.

- For speed reasons, the zero-crossing point of the loop function must be as well as possible translated towards the higher possible frequency.

A last remark is done for the component's values: as cut-off frequencies of the open loop are extremely low, it requires high resistances and capacitance values.

\subsubsection{Corrector schematic}

There is no unique schematic for the design of corrector stage $C(p)$. From \$7.2.1, we build the required corrector and we give in figure 21 below, the structure tested on our half quadricopter.

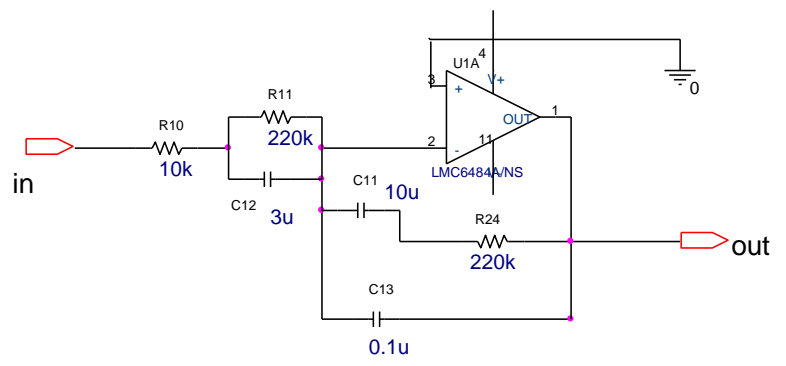

Figure 21: P.I.D Corrector schematic

For a visual checking of correction impact, adjustable components are placed on the electronic board

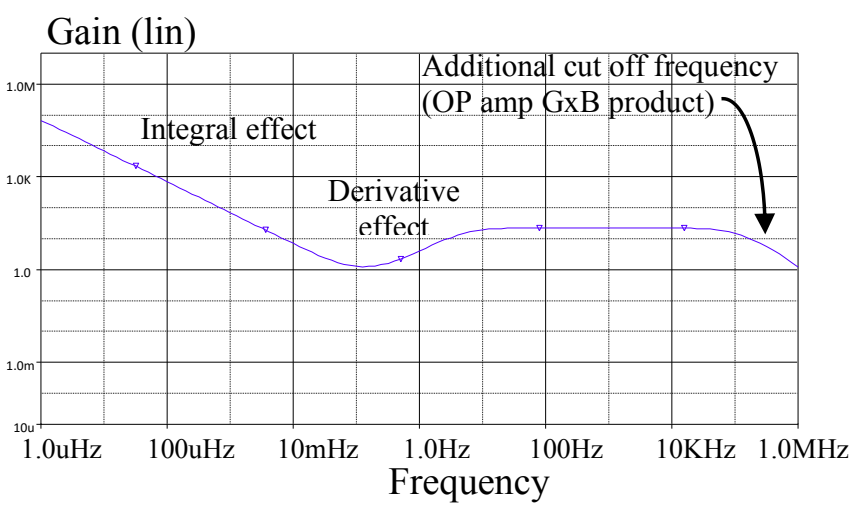

Figure 22: Correction AC response 


\subsubsection{Corrected open loop response}

Open loop AC response after correction looks like indicated in figure 23 . It is now ready to study the closed loop response.

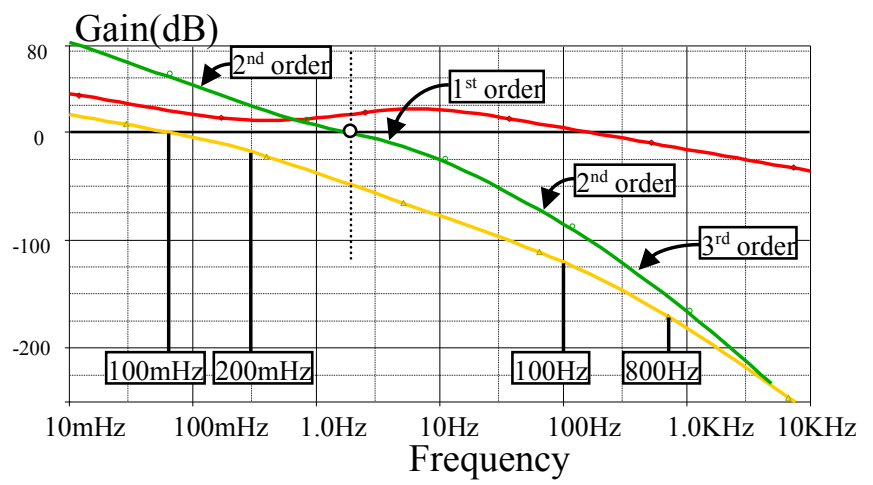

Figure 23: Open loop response

Yellow curve: open loop without correction.

Red curve: corrector P.I.D

Green curve: open loop gain after correction.

\subsection{Closed loop schematic and simulation}

The closed loop schematic is obtained from open loop diagram in figure 19, by adding "comparison to set up value" block, PID corrector, and closing the loop. DC, AC and transient stimuli are placed on set up voltage value, and each disturbance input.

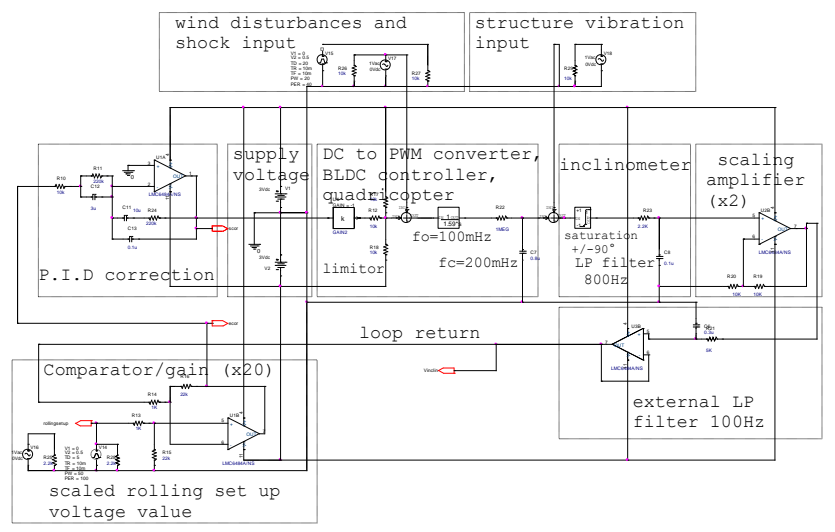

Figure 24: Closed loop schematic with PID correction (Orcad software).

\subsection{Closed loop response}

Figure 25 shows the simulated AC closed loop response $\operatorname{Vr}(\mathrm{p}) / \mathrm{Ve}(\mathrm{p})$. Bandwidth is around $2 \mathrm{~Hz}$ and a small resonance is observed.

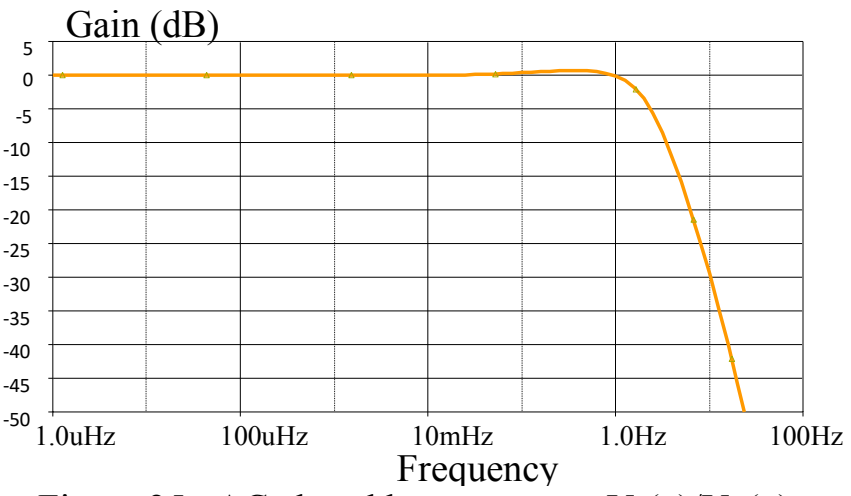

Figure 25: AC closed loop response $\operatorname{Vr}(\mathrm{p}) / \mathrm{Ve}(\mathrm{p})$

Figure 26 shows the closed loop simulated transient response to a set up control voltage pulse

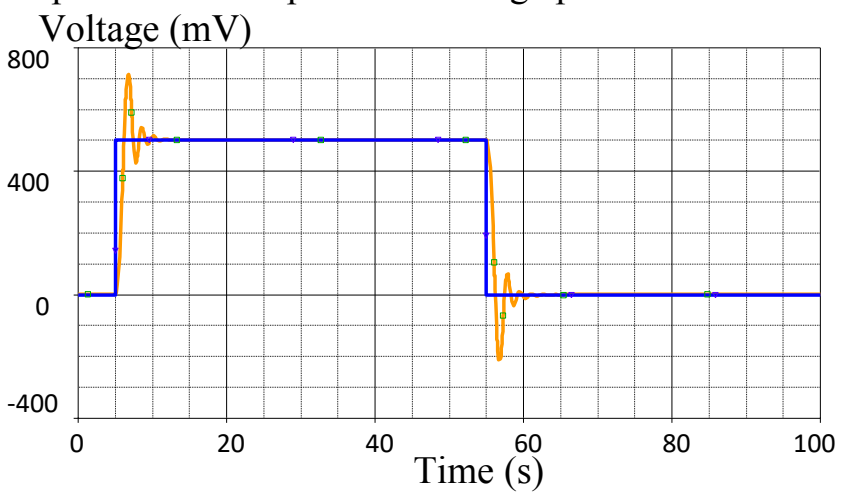

Figure 26: Closed loop response to rolling set up control signal pulse: $\operatorname{Vr}(\mathrm{t}) \mathrm{vs} . \mathrm{Ve}(\mathrm{t})$

Blue curve: set up voltage value Ve (rolling axis) Orange curve: returned voltage $\mathrm{Vr}$ (inclinometer output (with correction)

Scaling factor between Ve (in Volts) and inclination angle $\Theta$ (in degrees) is as follow: $0 \mathrm{Vdc}=>0^{\circ}$; $0.4 \mathrm{~V}=>14^{\circ} ; 1,6 \mathrm{~V}=>90^{\circ}$

\subsection{Effect of perturbations.}

Figure 27 shows the loop response when submitted to a pulse on $\mathrm{W}$ parasitic input. Impact of disturbance is greatly reduced: $\mathrm{Vr}$ return correctly to its initial value in less than $5 \mathrm{~s}$.

Red curve: pulse external perturbation on $\mathrm{W}$ input Green curve: returned value $\mathrm{Vr}$ by inclinometer (angle image) 


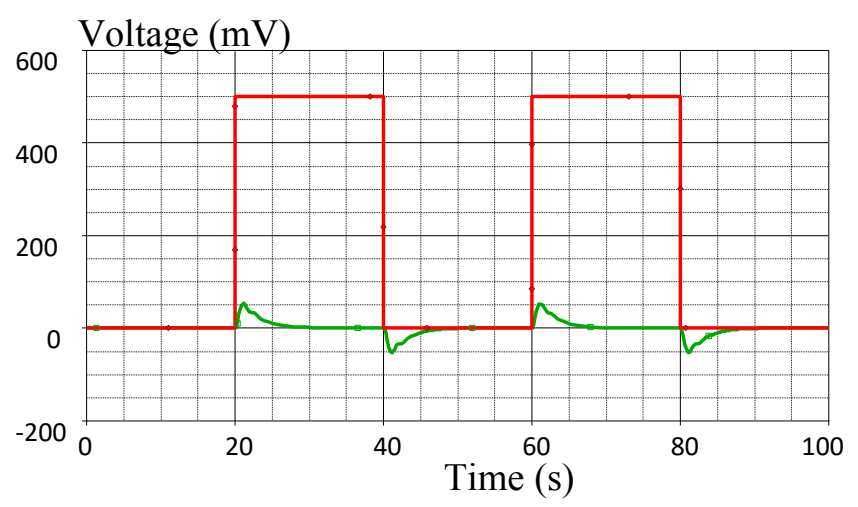

Figure 27: Closed loop response to a disturbance on $\mathrm{W}$ input

Figure 28 give the AC response of the loop to W input. According to the transfer function formula (3), it looks like a first order high pass in low frequencies et a first low pass in "high" frequencies.

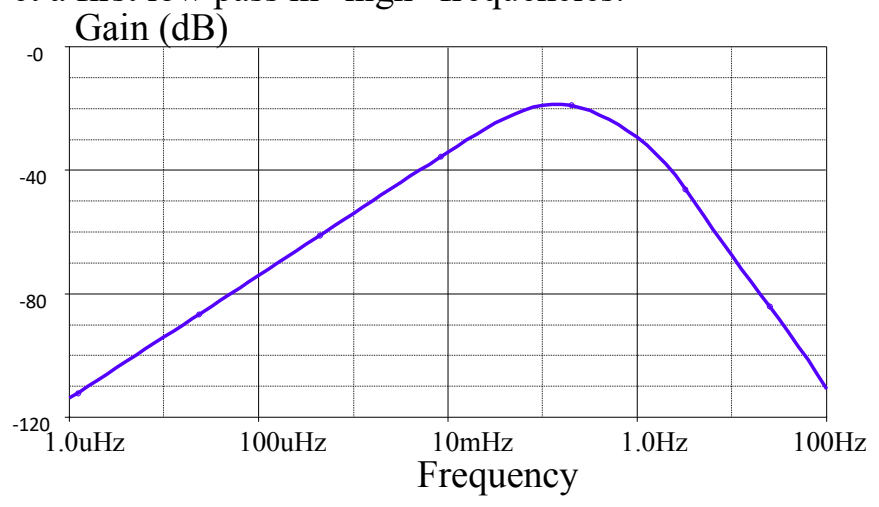

Figure 28: AC closed loop response $\operatorname{Vr}(\mathrm{p}) / \mathrm{W}(\mathrm{p})$ (disturbance on "W" input)

From figure 27and 28, it appears that a permanent disturbance (for example weight mismatching between the two arm extremities) can be compensated.

Figure 29 shows the AC response $\operatorname{Vr}(\mathrm{p}) / \operatorname{Pv}(\mathrm{p})$ of the closed loop to parasitic vibrations (Pv input).

$1,2 \mathrm{kHz}$ wood frame vibrations are rejected (around) $20 \mathrm{~dB}$ ) as well as low frequency disturbances. Possible disturbance between 1 to $100 \mathrm{~Hz}$ are not filtered. However, they do not exist fortunately on our quadricopter.

According to the transfer function equation (3) Behaviour looks like a second order in low frequency and first order low pass in "high" frequencies (after $100 \mathrm{~Hz}$ due to the cut off frequency of A3(p) block.

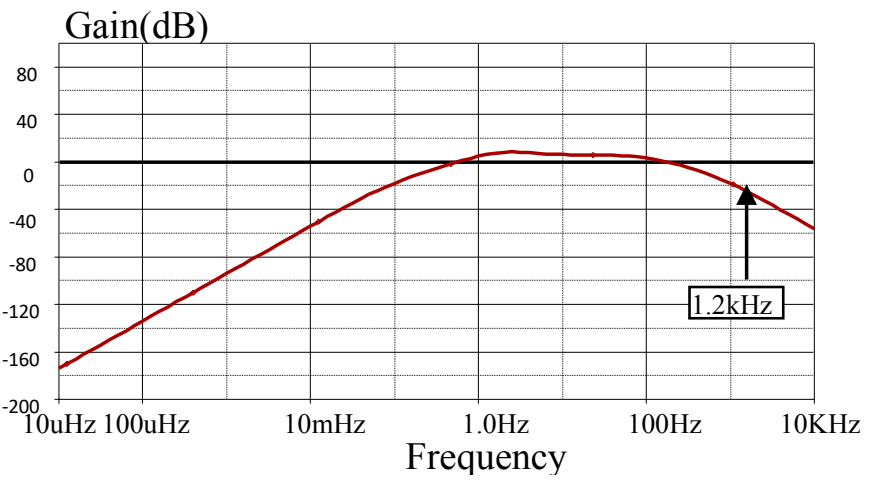

Figure 29: AC closed loop response $\operatorname{Vr}(\mathrm{p}) / \mathrm{Pv}(\mathrm{p})$ (disturbance on "Pv" input)

\subsection{Simulations results analysis and comments.}

From these simulations, we can predict a correct behaviour of the half quadricopter. However, we have to mention that adjustment of P.I.D correction parameters is very fine and sensitive and that non linearity's of air, turbulences due to ground effects are not modelled. Thus, simulations give a first global overview, but experimental adjustments might be required.

\section{Experimental tests}

Thus, we performed some visual tests to observe the behaviour of half quadricopter. Indeed, such practical approach is complementary to the previous simulation studies and allows a first technical assessment and comparison with simulations. Seeing effects of remaining defaults forces also to an intellectual analysis of phenomenon and oblige the students to a more sensitive and intuitive approach of the stability concepts.

\subsection{Electrical test}

Typical waveforms on 3 phases BLDC motors controller have been checked. Switched current and voltage waveforms across the 3 phases BLDC motors were measured.

The figure 30 shows typical waveform we obtained. 


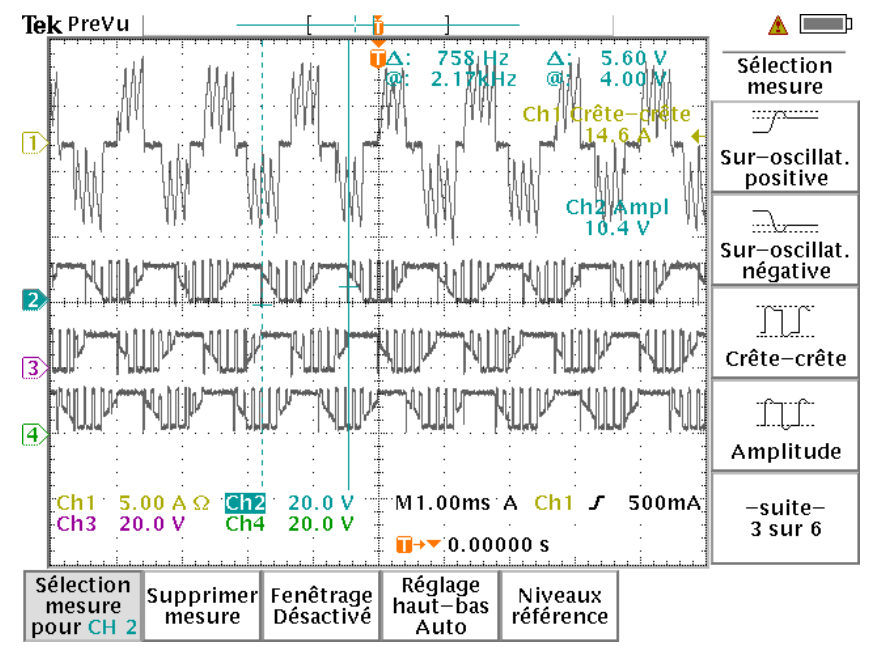

Figure 30: experimental voltage and current waveforms through the BLDC motor controller board

Trace 1 : Current chopped through the BLDC motor (scale: $5 \mathrm{~A} / \mathrm{div}$ )

Trace 2,3,4 : voltages on phase 1,2,3 PWM signal scale $(20 \mathrm{~V} / \mathrm{div})$

\subsection{Dynamic tests}

The global half quadricopter behaviour was tested visually as indicated hereafter. For each test, a video clip has been performed to record theses behaviours in order to make possible future comparisons. Figure 31 shows the experimental test bench.

\subsubsection{Response to the rolling set up control value}

Vertical push is set up at a medium value (i.e. control voltage $2.5 \mathrm{~V}$ DC to be in the middle of the range). Rolling set up control value is set up at 2.5VDC (i.e. quadricopter's arm horizontal).

Correct "quasi static" tracking of the rolling set up value is checked by modifying slowly the rolling set up value (with a potentiometer) to change angle inclination roughly from + to $-15^{\circ}$ and measuring the inclinometer output voltage.

Then, an external pulse is superposed to the rolling set up value to show the transient response. A response with damped oscillations is observed according to simulation in figure 26 .

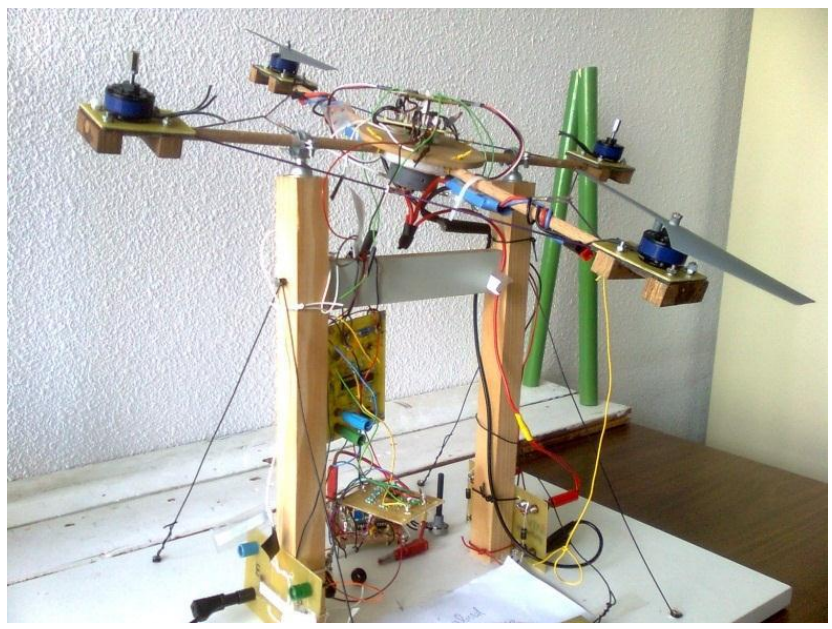

Figure 31: Experimental platform

\subsubsection{Effects of disturbances}

Two kinds of experiences were done:

1) Small calibrated weights (30grs and 50grs and $100 \mathrm{grs})$ are hanged under one the motors causing a permanent weight difference between the arm extremities.

Visible change in rotation speed of the propeller is observed: It is automatically increased by the loop, above the heaviest side. Balance is correct for the 30 grs ballast. With the 50grs ballast, a small permanent error of a few degrees is observed. With 100 grs ballast, motor and propeller are no more enough powerful to balance the quadricopter: Indeed, from vertical thrust characterisation in $\$ 4.2 .1$, the maximum push is "only" 280 grs per propeller.

2) A small mechanical shock is applied with a small stick under one extremity of the quadricopter's arm to simulate a sudden impact. The half quadricopter return correctly to equilibrium after a relaxation time like predicted in figure 27.

\subsection{Understanding impact of non linearity in the loop}

\subsubsection{Visual test}

Visual test shows that stability is quite correct at medium vertical thrust level. At low and high-power level, oscillations appear and quadricopter becomes unstable. Indeed, due to the nonlinear shape of the response curve in figure 10, variations of thrust at 
these two extreme levels, is not enough significant to make a reliable balance of the quadricopter arm.

\subsubsection{Linearity correction}

Thus, linearity compensation is required to get the same behaviour whatever the power thrust level. A simple circuit with OP amp LMC6482 and two diodes (figure 32a) has been added.

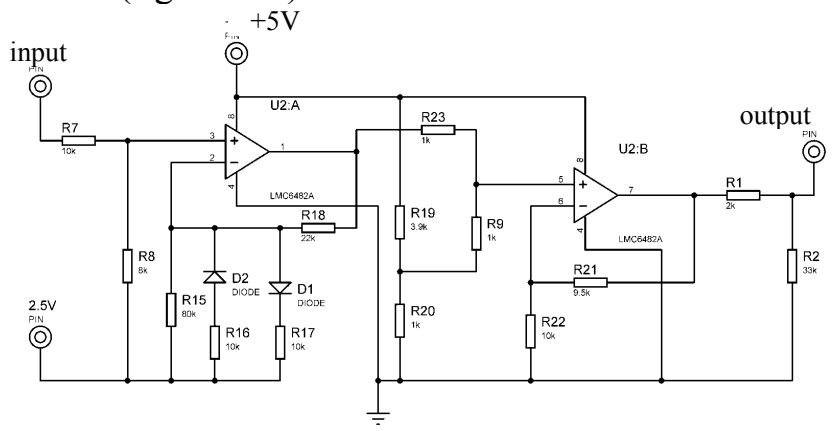

Figure 32a: Linearization circuit

Its DC transfer function (cf. figure $32 \mathrm{~b}$ ) is built to compensate the non-linearity of figure 10 .

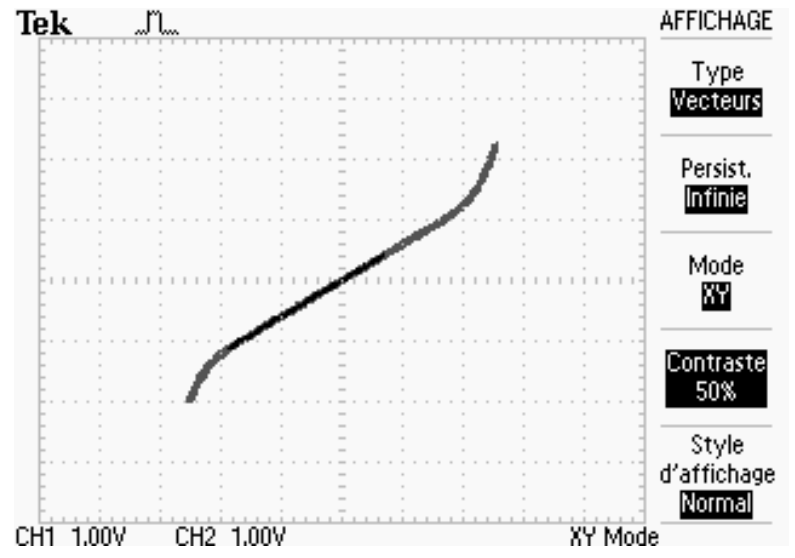

Figure 32b: Corresponding DC transfer function (oscilloscope in XY mode)

Thus, association of the two transfer functions (figure $32 \mathrm{~b}$ and 10) gives a global linear transfer function over the full range. New tests at various vertical thrust levels are now satisfying.

\section{Discussion}

As told previously, this study is first of all, a practical approach of a complex problem. Our system is obviously not able to compete with fully digital ultra- sophisticated existing leisure or professional quadricopters: Precise flight stabilization or acrobatic flights were out of the subject. However, presented results are satisfying and enough significant to understand impact of feedback control and various aspect of loop design (gain, stability, non-linear aspects...). Technical improvements should be, of course, numerous and various: for example, we must work again on compensation of permanent weight asymmetry between the arm extremities which is not enough satisfying till now.

\section{Study assessment}

Merging experiment, modelling extraction and simulations and visual tests allowed us to learn about the physical behaviour of UAV system and fine impact and each electronic block.

We plan to goes on the project by building the electronic loop for second arm (similar to this first one).

Once finished, lace control loop study including gyroscope module will start. Then, main difficulty will be the intrinsic coupling between the flying parameters control.

\section{Conclusion}

A complex analogue electronic feed back was successfully studied, to balance a half quadricopter. A good matching between simulations and experimental results has been shown. Despite the complexity of the system, interesting results were obtained without heavy mathematical tools.

Learning from practical experiences looks like a complementary approach to traditional theoretical approaches. It seems to be especially suitable for "right brain" people who need an intuitive rather than a pure mathematical way of minding.

References:

[1] Parrot french company web site : https://www.parrot.com/fr/drones/anafi

[2] K. Buyukkabasakal, B. Fidan and A. Savran, "Mixing adaptive fault tolerant control of quadrotor UAV," Asian Journal of Control, vol. 19, no. 4, pp. 1441-1454, July 2017.

[3] N. Koksal, H. An and B. Fidan, "Two-Level Nonlinear Tracking Control of a Quadrotor 
Unmanned Aerial Vehicle" in Proc. IFACPapersOnLine, vol. 49, no. 17, pp. 254-259, 2016. [4] C. Izaguirre-Espinosa, A.J. Munoz-Vazquez, A. Sanchez-Orta, V. Parra-Vega and P. Castillo, "Attitude control of quadrotors based on fractional sliding modes: theory and experiments" IET Control Theory Applications, vol. 10, no. 7, pp. 825-832, 2016.

[5] M. Hedayatpour, M. Mehrandezh and F. JanabiSharifi, "A unified approach to configuration based dynamic analysis of quadcopters for optimal stability" Int. Conference on Intelligent Robots and Systems (IROS), pp. 5116-5121, Vancouver, BC, Canada, September 2017.

[6] Corentin CHAUFFAUT, "Étude de la phase de transition d'un drone tiré par tube dédié : modélisation et commande" Thèse soutenue le 07 octobre 2014 UTC compiègne France

[7] P. Kettle, A. Murray-F. Moynihan. "Sensorless Control of a Brushless DC motor using an Extended" Kalman estimator. PCIM'98 INTELLIGENT MOTION. May 1998 PROCEEDINGS pp 385. 392

WSEAS EDUTE '07 23-25 novembre 2007 Venise (Italie)

[8] Ph. Dondon, J.M Micouleau, M.Marthiens Dagorette "A practical and integrated approach for power switching supply teaching: example of a BOOST converter topology" WSEAS EDUTE '07 2325 novembre 2007 Venise (Italie)

[9] A. Serrecourt- J. Chapuis «Stabilisation d'un quadrirotor » rapport ENSICA 2006-2007

[10] N. MIRJOLET-A. ROUSSEL «Conception d'une aide au pilotage pour un quadricoptère" rapport SUPELEC Avril 2004

[11] A.Frenot - A.Gossmann-R. Guillerm

"Stabilisation d'un quadrirotor" rapport ENSICA 2005-2006

[12] T.Hamel, MR. Mahony, R Lozano, J. Oststrowski "Dynamic modelling and configuration stabilisation for an X4-flyer" International Federation of automatic control symposium, IFAC 2002 Barcelona, Spain

[13] T. L. Grigorie, N.Jula, C. Cepisca, C. Răcuciu, D. răducanu "Evaluation Method of the Sensors Errors in an Inertial Navigation System" WSEAS TRANSACTIONS on CIRCUITS and SYSTEMS ISSN: 1109-2734 p977 Issue 12, Volume 7, December 2008

[14] S. Punpaisarn, S. Sujitjorn “ SUT-CARG CarLike Robots: Their Electronics and Control
Architecture" WSEAS TRANSACTIONS on CIRCUITS AND SYSTEMS ISSN: 1109-2734 p579 Issue 6, Volume 7, June 2008

[15] T. L. Grigorie, N.Jula, C. Cepisca, C. Răcuciu, D. Răducanu "MEMS based Micro inertial measurement system" WSEAS TRANSACTIONS on CIRCUITS and SYSTEMS ISSN: 1109-2734 p691 Issue 5, Volume 5, May 2006

[16]MMA7260Q micromachined accelerometer data sheet web site Freescale Semiconductor

[17] P. Yedamale "using PIC 18F2431 for sensorless BLDC motor control" Application note $\mathrm{N}^{\circ} 970$ Microchip

[18] P. Castillo,-R. Lozano-A. Dzul, "Modelling and Control of Mini-Flying Machines", Springer-Verlag in Advances in Industrial Control, July 2005.

[19] F.Jensen, D. R. Hagen Pedersen "Autonomous Aircraft, a nonlinear approach" Group 1034 IAS10 2005 - Aalborg University

[20] B. Cazzolato "Automatic Control II - 2DOF Helicopter Tutorial \& Lab” September 2006 